\title{
Blow-up for Discretizations of a Nonlinear Parabolic Equation With Nonlinear Memory and Mixt Boundary Condition
}

\author{
Camara Zié ${ }^{1}$, N'gohisse Konan Firmin ${ }^{2}$ \& Yoro Gozo ${ }^{1}$ \\ ${ }^{1}$ Université Nangui Abrogoua, UFR-SFA, Département de Mathématiques et Informatiques, Côte d'Ivoire \\ ${ }^{2}$ Université Péléforo Gon Coulibaly de Korhogo, Département de Mathématiques et Informatiques, Côte d'Ivoire \\ Correspondence: Camara Zié, Université Nangui Abrogoua, UFR-SFA, Département de Mathématiques et Informatiques, \\ 14 BP 1588 Abidjan 14, Côte d'Ivoire. E-mail: ziecamara892@yahoo.fr
}

Received: September 22, 2019 Accepted: October 25, 2019 Online Published: November 4, 2019

doi:10.5539/jmr.v11n6p29

URL: https://doi.org/10.5539/jmr.v11n6p29

\section{Abstract}

In this paper, we study the numerical approximation for the following initial-boundary value problem

$$
\left\{\begin{array}{l}
v_{t}=v_{x x}+v^{q} \int_{0}^{t} v^{p}(x, s) d s, x \in(0,1), t \in(0, T), \\
v(0, t)=0, v_{x}(1, t)=0, t \in(0, T), \\
v(x, 0)=v_{0}(x)>0, x \in(0,1),
\end{array}\right.
$$

where $q>1, p>0$. Under some assumptions, it is shown that the solution of a semi-discrete form of this problem blows up in the finite time and estimate its semi-discrete blow-up time. We also prove that the semi-discrete blows-up time converges to the real one when the mesh size goes to zero. A similar study has been also undertaken for a discrete form of the above problem. Finally, we give some numerical results to illustrate our analysis.

Keywords: semi-discretization, nonlinear parabolic equation, blow-up, numerical blow-up time, nonlinear memory, finite difference, discretization

\section{Introduction}

Consider the following problem

$$
\begin{gathered}
v_{t}=v_{x x}+v^{q} \int_{0}^{t} v^{p}(x, s) d s, x \in(0,1), t \in(0, T), \\
v(0, t)=0, v_{x}(1, t)=0, t \in(0, T), \\
v(x, 0)=v_{0}(x)>0, x \in(0,1),
\end{gathered}
$$

which models the temperature distribution of a large number of physical phenomenon from physics, chemistry and biology. In particular, the above problem has a lot of applications in the theory of nuclear reactor kinetics see (Kozhanov, 1994 for more physical motivations). The initial datum $v_{0}(x)$ is a continuous function in $(0,1), v_{0}(0)=0, v_{x}(1)=0, q>1$, $p>0$. The conditions $v_{0}(0, t)=0$ means that the temperature is maintained nil on the boundary $x=0$. Here $(0, T)$ is the maximal time interval on which the solution $v$ of (1)-(3) exists. The time $T$ may be finite or infinite. When $T$ is infinite, we say that the solution $u$ exists globally. When $T$ is finite, the solution $u$ develops a singularity in a finite time, namely

$$
\lim _{t \rightarrow T}\|v(\cdot, t)\|_{\infty}=\infty
$$

where $\|v(\cdot, t)\|_{\infty}=\max _{0 \leq x \leq 1}|v(x, t)|$. In this case, we say that the solution $v$ blows up in a finite time and the time $T$ is called the blow-up time of solution $v$. Solutions of nonlinear parabolic equations which blow up in finite time have been the subject of investigations of many authors see (Brandle et al., 2005; Galaktionov et al., 2002; Groisman, 2006; Hirata, 1999; N'gohisse and Boni, 2008 and the references cited therein). In particular, in (Galaktionov et al., 2002; Groisman et al., 2004; Hirata, 1999; Koffi and Nabongo, 2016; Li, 2009; Quittner and Souplet, 2007; Sobo et al., 2016; Souplet, 2004; Zhang et al., 2010; Zhou, 2007), the above problem has been considered and existence and uniqueness of a classical solution have been proved. Under some assumptions, the authors have also shown that the classical solution blows up in a finite time and its blow-up time has been estimated. 
The aim of this paper is the numerical study of the above problem.

Let $I$ be a positive integer and define the grid $x_{i}=i h, 0 \leq i \leq I$, where $h=\frac{1}{I}$. Approximate the solution $v$ of the problem (1)-(3) by the solution $V_{h}(t)=\left(V_{0}(t), V_{1}(t), \ldots, V_{I}(t)\right)^{T}$ of the following semi-discrete equations

$$
\begin{gathered}
\frac{d V_{i}(t)}{d t}=\delta^{2} V_{i}(t)+V_{i}^{q}(t) \int_{0}^{t} V_{i}^{p}(s) d s, 1 \leq i \leq I, t \in\left(0, T_{b}^{h}\right), \\
V_{0}(t)=0, \\
V_{i}(0)=\varphi_{i}>0,0 \leq i \leq I, \\
\varphi_{i+1}>\varphi_{i}, \quad 0 \leq i \leq I-1,
\end{gathered}
$$

where

$$
\begin{gathered}
\delta^{2} V_{i}(t)=\frac{V_{i+1}(t)-2 V_{i}(t)+V_{i-1}(t)}{h^{2}}, \quad 1 \leq i \leq I-1, \\
\delta^{2} V_{I}(t)=\frac{2 V_{I-1}(t)-2 V_{I}(t)}{h^{2}} .
\end{gathered}
$$

Here, $\left(0, T_{b}^{h}\right)$ is the maximal time interval on which $\|v(., t)\|_{\infty}$ is finite, where $\|v(., t)\|_{\infty}=\max _{0 \leq x \leq 1}|v(x, t)|$. When $T_{b}^{h}$ is finite, we say that the solution $V_{h}(t)$ of (4)-(6) blows up in the finite time and the time $T_{b}^{h}$ is called the semi-discrete blow-up time of the solution $V_{h}(t)$.

Abia et al., (1998) have considered the equation (1)-(3) in the case where the source $v^{q} \int_{0}^{t} v^{p}(x, s) d s$ is replaced by $v^{p}$. They have considered a scheme as the one given in (4)-(6). They have shown that the semi-discrete solution blows up in the finite time and its blow-up time goes to the real one when the mesh size tends to zero.

In this paper, firstly, we show that under some assumptions, the solution of the semi-discrete problem defined in (4)-(6) blows up in a finite time and estimate its semi-discrete blow-up time. We also show that the semi-discrete blow-up time converges to the real one when the mesh size goes to zero. In addition we give the blow-up rate of the solution of the semi-discrete problem. A similar study has been also undertaken for a full discrete form of (1)-(3). Let us notice that in (Abia et al.,1998), only the semi-discrete scheme has been analyzed. One may find in (Mai et al., 1991; Brandle et al., 2004; Ferreira et al., 2004; Li and Xie, 2004; Kozhanov, 1994; N'gohisse and Boni, 2011; Pablo and al, 2005), similar studies concerning other parabolic problems. Let us notice that many authors have used numerical methods to study the phenomenon of blow-up but they are only a few studies on the convergence of the numerical blows-up time for solutions which blow-up in $L^{\infty}$ norm. For instance in (Groisman, 2006), the authors have proved the convergence of numerical blow-up time for solutions which blow up in $L^{p}$ norm with $1<p<\infty$.

The rest of the paper is organized as follows. In the next section, we give some results which will be used later. In the section 3, under some conditions, we prove that the solution of the semi-discrete problem blows up in a finite time and estimate its semi-discrete blow-up time. In the fourth section, we show that, under some additional hypothesis, the semi-discrete blow-up time goes to the real one when the mesh size goes to zero. In the fifth section, we obtain similar results as in sections 3 and 4 using a discrete scheme. Finally, in the last section we report on some numerical experiments to illustrate our analysis.

\section{Properties of the Semi-discrete Problem}

In this section, we give some results which will be used later. The following lemma is a semi-discrete form of the maximum principle.

Lemma 1 Let $a_{h} \in C^{0}\left([0, T], \mathbb{R}^{I+1}\right)$ and let $W_{h} \in C^{1}\left([0, T], \mathbb{R}^{I+1}\right)$ be such that

$$
\begin{gathered}
\frac{d W_{i}(t)}{d t}-\delta^{2} W_{i}(t)+a_{i}(t) W_{i}(t) \geq 0,1 \leq i \leq I, t \in(0, T), \\
W_{0}(t) \geq 0, t \in(0, T) \\
W_{i}(0) \geq 0,0 \leq i \leq I .
\end{gathered}
$$

Then we have $W_{i}(t) \geq 0,0 \leq i \leq I, t \in(0, T)$.

Proof. For the proof, see (N'gohisse and Boni, 2011).

The semi-discrete form of the comparison lemma is staded as follow. 
Lemma 2 Let $f \in C^{0}(\mathbb{R} \times \mathbb{R}, \mathbb{R})$ and let $W_{h}, X_{h} \in C^{1}\left([0, T], \mathbb{R}^{I+1}\right)$ be such that for $t \in(0, T)$

$$
\begin{aligned}
\frac{d W_{i}(t)}{d t}-\delta^{2} W_{i}(t)+f\left(W_{i}(t), t\right) & >\frac{d X_{i}(t)}{d t}-\delta^{2} X_{i}(t)+f\left(X_{i}(t), t\right), \quad 1 \leq i \leq I, \\
& W_{0}(t)>X_{0}(t), \\
W_{i}(0) & >X_{i}(0), \quad 0 \leq i \leq I .
\end{aligned}
$$

Then we have $W_{i}(t)>X_{i}(t), 0 \leq i \leq I, t \in(0, T)$.

Proof. See (N'gohisse and Boni, 2011) for the proof.

The lemma below shows the positivity of the solution.

Lemma 3 Let $V_{h}$ be the solution of (4)-(6). Then we have

$$
V_{i}(t)>0,0 \leq i \leq I, t \in\left(0, T_{b}^{h}\right) .
$$

Proof. From Lemma $1, V_{h}(t) \geq 0$ for $t \in\left(0, T_{b}^{h}\right)$. Suppose that there exist $i_{0} \in\{1, \ldots, I\}$ and $t_{0} \in\left(0, T_{b}^{h}\right)$ such that $V_{i_{0}}\left(t_{0}\right)=0$. We observe that $\frac{d V_{i_{0}}\left(t_{0}\right)}{d t} \leq 0$ and $\delta^{2} V_{i_{0}}\left(t_{0}\right) \geq 0$. We deduce that

$$
\frac{d V_{i_{0}}\left(t_{0}\right)}{d t}-\delta^{2} V_{i_{0}}\left(t_{0}\right)+V_{i}^{q}\left(t_{0}\right) \int_{0}^{t} V_{i}^{p}(s) d s<0 .
$$

But this contradicts (4) and we have the desidered result.

Lemma 4 Let $V_{h}$ be the solution of (4)-(6). Then we have

$$
V_{i+1}(t)>V_{i}(t), 1 \leq i \leq I-1, t \in\left(0, T_{b}^{h}\right) .
$$

Proof. Let $Y_{i}(t)=V_{i+1}(t)-V_{i}(t)$, for $0 \leq i \leq I-1$. Since from Lemma $3 \quad V_{1}>0$ for $t \in\left(0, T_{b}^{h}\right)$, we get $Y_{0}(t)>0$ for $t \in\left(0, T_{b}^{h}\right)$. Let $t_{0}$ be the first $t \in\left(0, T_{b}^{h}\right)$ such that $Y_{i}\left(t_{0}\right)>0$ for $t \in\left(0, t_{0}\right), 1 \leq i \leq I-1$, but $Y_{0}(t)=0$ for a certain $i_{0} \in\{1, \ldots, I-1\}$. Without less of generality, we may suppose that $i_{0}$ is the smallest $i$ which satisfies the equality. We observe that

$$
\begin{gathered}
\frac{d Y_{i_{0}}\left(t_{0}\right)}{d t}=\lim _{k \rightarrow 0} \frac{Y_{i_{0}}\left(t_{0}\right)-Y_{i_{0}}\left(t_{0}-k\right)}{k} \leq 0, \\
\delta^{2} Y_{i_{0}}\left(t_{0}\right)=\frac{Y_{i_{0}+1}\left(t_{0}\right)-2 Y_{i_{0}}\left(t_{0}\right)+Y_{i_{0}-1}\left(t_{0}\right)}{h^{2}}>0, \quad \text { if } 1 \leq i_{0} \leq I-2, \\
\delta^{2} Y_{i_{0}}\left(t_{0}\right)=\frac{-3 Y_{I-1}\left(t_{0}\right)+2 Y_{I-2}\left(t_{0}\right)}{h^{2}}>0 \quad \text { if } i_{0}=I-1 .
\end{gathered}
$$

We deduce that

$$
\frac{d Y_{i_{0}}\left(t_{0}\right)}{d t}-\delta^{2} Y_{i_{0}}\left(t_{0}\right)+Y_{i_{0}}^{q}\left(t_{0}\right) \int_{0}^{t} Y_{i}^{p}(s) d s<0, \quad \text { if } 1 \leq i_{0} \leq I .
$$

But this contradicts (4) and the proof is complete.

The following result reveals the property of the operator $\delta^{2}$.

Lemma 5 Let $V_{h} \in \mathbb{R}^{I+1}$ such that $V_{h} \geq 0$. Then we have

$$
\delta^{2} V_{i}^{p} \geq p V_{i}^{p-1} \delta^{2} V_{i}, \quad 1 \leq i \leq I .
$$

Proof. See (N'gohisse and Boni, 2011).

Lemma 6 Let $W_{h}$ and $V_{h} \in \mathbb{R}^{I+1}$. If $\delta^{-}\left(V_{I}\right) \delta^{-}\left(W_{I}\right) \geq 0$ and

$$
\delta^{+}\left(V_{i}\right) \delta^{+}\left(W_{i}\right) \geq 0, \delta^{-}\left(V_{i}\right) \delta^{-}\left(W_{i}\right) \geq 0, \quad 1 \leq i \leq I-1,
$$

then

$$
\delta^{2}\left(V_{i} W_{i}\right) \geq V_{i} \delta^{2} W_{i}+W_{i} \delta^{2} V_{i}, \quad 1 \leq i \leq I,
$$

where $\delta^{+}\left(V_{i}\right)=\frac{V_{i+1}-V_{i}}{h}$ and $\delta^{-}\left(V_{i}\right)=\frac{V_{i-1}-V_{i}}{h}$. 
Proof. See (N'gohisse and Boni, 2011).

\section{Blow-up in the Semi-discrete Problem}

In this section under some conditions, we prove that the solution $V_{h}$ of (4)-(6) blows up in a finite time and estimate its semi-discrete blow-up time. Our first result on the blow-up is the following.

Theorem 1 Let $V_{h}$ be the solution of (4)-(6) and suppose that there exists a positive constant $A \in(0,1]$ such that the initial datum at (6) satisfies

$$
\delta^{2} \varphi_{i}+\varphi_{i}^{q} \geq A \sin \left(i h \frac{\pi}{2}\right) \varphi_{i}^{q}, \quad 1 \leq i \leq I .
$$

Then the solution $V_{h}$ blows-up in a finite time $T_{b}^{h}$ which is estimated as follows

$$
T_{b}^{h} \leq \frac{2}{\pi^{2}} \ln \left(1-\frac{\pi^{2}}{2 A} \frac{\left\|\varphi_{I}\right\|_{\infty}^{1-q}}{(1-q)}\right) .
$$

Proof. Let $T_{b}^{h}$ be the time up to which $\left\|V_{h}(t)\right\|_{\infty}$ is finite. Our aim is to show that $T_{b}^{h}$ is finite and obeys the above inequality. Introduce the vector $J_{h}$ defined as follows

$$
J_{i}(t)=\frac{d V_{i}(t)}{d t}-C_{i}(t) V_{i}^{q}(t), \quad 0 \leq i \leq I, \quad t \in\left(0, T_{b}^{h}\right),
$$

where $C_{i}(t)=A e^{-\lambda_{h} t} \sin \left(i h \frac{\pi}{2}\right), \quad 0 \leq i \leq I, t \in\left(0, T_{b}^{h}\right)$, with $\lambda_{h}=\frac{2-2 \cos \left(i h \frac{\pi}{2}\right)}{h^{2}}$.

A routine computation reveals that

$$
\frac{d J_{i}}{d t}-\delta^{2} J_{i}=\frac{d}{d t}\left(\frac{d V_{i}}{d t}-\delta^{2} V_{i}\right)-C_{i}(t) q V_{i}^{q-1} \frac{d V_{i}}{d t}+\delta^{2}\left(C_{i} V_{i}^{q}\right), \quad 1 \leq i \leq I .
$$

We observe that

$$
\frac{d C_{i}}{d t}-\delta^{2} C_{i}=0, C_{i-1}<C_{i}, \quad 1 \leq i \leq I,
$$

and due to Lemma 4 we find that

$$
\delta^{-}\left(V_{I}^{q}\right) \delta^{-}\left(C_{I}\right) \geq 0, \delta^{+}\left(V_{i}^{q}\right) \delta^{+}\left(C_{i}\right) \geq 0
$$

and

$$
\delta^{-}\left(V_{i}^{q}\right) \delta^{-}\left(C_{i}\right) \geq 0, \quad 1 \leq i \leq I-1 .
$$

From Lemma 5 and Lemma 6, we get

$$
\delta^{2}\left(C_{i}(t) V_{i}^{q}(t)\right) \geq C_{i}(t) q V_{i}^{q-1}(t) \delta^{2} V_{i}(t)+V_{i}^{q}(t) \delta^{2} C_{i}(t), \quad 1 \leq i \leq I .
$$

Using the above estimates, we discover that

$$
\frac{d J_{i}}{d t}-\delta^{2} J_{i} \geq \frac{d}{d t}\left(\frac{d V_{i}}{d t}-\delta^{2} V_{i}\right)-C_{i} q V_{i}^{q-1}\left(\frac{d V_{i}}{d t}-\delta^{2} V_{i}^{q}\right)+V_{i}^{q}\left(\frac{d C_{i}}{d t}-\delta^{2} C_{i}\right), \quad 1 \leq i \leq I .
$$

With the help of (4), we obtain for $1 \leq i \leq I$ that

$$
\frac{d J_{i}}{d t}-\delta^{2} J_{i} \geq q V_{i}^{q-1} \frac{d V_{i}}{d t}-C_{i} q V_{i}^{q-1}\left(V_{i}^{q} \int_{0}^{t} V_{i}^{p}(s) d s\right)
$$

Due the fact that $\frac{d V_{i}}{d t}=J_{i}(t)+C_{i} V_{i}^{q}$, we arrive at

$$
\frac{d J_{i}}{d t}-\delta^{2} J_{i} \geq\left(q V_{i}^{q-1} \frac{d V_{i}}{d t} \int_{0}^{t} V_{i}^{p}(s) d s\right) J_{i}, \quad 1 \leq i \leq I, t \in\left(0, T_{b}^{h}\right)
$$

Obviously, we have $J_{0}(t)=0$, and $J_{h}(0) \geq 0$ because of (7). We deduce from Lemma 1 that

$$
J_{h}(t) \geq 0 \text { for } t \in\left(0, T_{b}^{h}\right)
$$


Which implies that

$$
\frac{d V_{I}}{d t}-C_{I} V_{I}^{q} \geq 0, \quad t \in\left(0, T_{b}^{h}\right) .
$$

This estimation may be rewritten in the following form

$$
\frac{d V_{I}}{V_{I}^{q}} \geq A e^{-\lambda_{h} t} d t
$$

Applying Taylor's expansion to obtain

$$
\cos \left(\frac{\pi h}{2}\right)=1-\frac{\pi^{2} h^{2}}{4}+\frac{\pi^{3} h^{3}}{48} \sin \left(\frac{\pi h}{2} \theta\right) \text { where } \theta \in[0,1], \text { this implies that } \lambda_{h} \leq \frac{\pi^{2}}{2} .
$$

Therefore using (8), we discover that

$$
\frac{d V_{I}}{V_{I}^{q}} \geq A e^{-\frac{\pi^{2} t}{2}} d t, t \in\left(0, T_{b}^{h}\right) .
$$

Integrating this inequality over $\left(0, T_{b}^{h}\right)$, we obtain

$$
T_{b}^{h} \leq \frac{2}{\pi^{2}} \ln \left(1-\frac{\pi^{2}}{2 A} \frac{\left\|V_{I}(0)\right\|_{\infty}^{1-q}}{(1-q)}\right), t \in\left(0, T_{b}^{h}\right) .
$$

From Lemma 4, $\left\|V_{h}(t)\right\|_{\infty}=V_{I}(t)$. Use the fact that $V_{I}(0)=\left\|\varphi_{h}\right\|_{\infty}$ to complete the rest of the proof.

Remark 1 Integrate the inequality (8) over $\left(t_{0}, T_{b}^{h}\right)$ to obtain

$$
T_{b}^{h}-t \leq \frac{2}{\pi^{2}} \ln \left(1-\frac{\pi^{2}}{2 A} e^{-\lambda_{I}} \frac{\left\|V_{h}(t)\right\|_{\infty}^{1-q}}{1-q}\right) \quad \text { for } t \in\left(0, T_{b}^{h}\right) .
$$

Since $\left\|V_{h}(t)\right\|_{\infty}=V_{I}(t)$ and $\lambda_{I}=0$ we get,

$$
T_{b}^{h}-t_{0} \leq \frac{2}{\pi^{2}} \ln \left(1-\frac{\pi^{2}}{2 A} \frac{V_{I}^{1-q}\left(t_{0}\right)}{1-q}\right) \text { for } t_{0} \in\left(0, T_{b}^{h}\right) .
$$

\section{Convergence of the Semi-discrete Blow-up Time}

Here, we show that the solution of the semi-discrete problem blows up in a finite time and its blows-up time goes to the continious one when the mesh size goes to zero. We denote

$$
v_{h}(t)=\left(v\left(x_{0}, t\right), \ldots, v\left(x_{I}, t\right)\right)^{T}, \quad\left\|V_{h}(t)\right\|_{\infty}=\max _{0 \leq i \leq I}\left|V_{i}(t)\right|
$$

and $C^{4,1}([0,1] \times[0, T])$ the space of function $k$-times continuously differentiable by report has $x$ in $[0,1] l$-times continuously differentiable by report has $t$ in $[0, T]$. In order to obtain the convergence of the semi-discrete blow-up time, we firstly prove the following theorem about the convergence of the semi-discrete scheme.

Theorem 2 Assume that the problem (1)-(3) has a solution $v \in C^{4,1}([0,1] \times[0, T])$ and the initial datum at (6) satisfies

$$
\left\|\varphi_{h}-v_{h}(0)\right\|_{\infty}=o(1) h \rightarrow 0 .
$$

Then for $h$ sufficiently small, the problem (4)-(6) has a unique solution $V_{h} \in C^{1}\left([0,1], \mathbb{R}^{l+1}\right)$ such that

$$
\max _{0 \leq i \leq I}\left\|V_{h}(t)-v_{h}(t)\right\|_{\infty}=O\left(\left\|\varphi_{h}-v_{h}(0)\right\|_{\infty}+h^{2}\right) \text { as } h \rightarrow 0 .
$$

Proof. Since $v \in C^{4,1}$, there exists a positive constant $K$ such that

$$
\frac{\left\|v_{x x x x}\right\|_{\infty}}{12} \leq K, \quad\|v\|_{\infty} \leq K .
$$

The problem (4)-(6) has for each $h$, a unique solution $V_{h} \in C^{1}\left(\left[0, T_{b}^{h}\right], \mathbb{R}^{l+1}\right)$. Let $t(h) \leq \min \left\{T, T_{b}^{h}\right\}$ the greatest value of $t>0$ such that

$$
\left\|V_{h}(t)-v_{h}(t)\right\|_{\infty}<1 \text {, for } t \in(0, t(h)) .
$$


The relation (9) implied that $t(h)>0$ for $h$ sufficiently small. By the triangle inequality, we obtain

$$
\left\|V_{h}(t)\right\|_{\infty} \leq\left\|v_{h}(t)\right\|_{\infty}+\left\|V_{h}(t)-v_{h}(t)\right\|_{\infty} \text { for } t \in(0, t(h)),
$$

which implies that

$$
\left\|V_{h}(t)\right\|_{\infty} \leq 1+K, \quad t \in(0, t(h)) .
$$

Since $v \in C^{4,1}$, taking the derivative in $x$ on both sides of (1) and due to the fact that $v_{x}$ and $v_{x t}$ vanish at $x=1$, we observe that $v_{x x x}$ vanishes at $x=1$. Applying Taylor's expansion, we discover that, for $1 \leq i \leq I-1, t \in(0, t(h))$,

$$
\begin{gathered}
v_{x x}\left(x_{i}, t\right)=\delta^{2} v\left(x_{i}, t\right)-\frac{h^{2}}{12} v_{x x x x}\left(x_{i}, t\right) . \\
v_{t}\left(x_{i}, t\right)-\delta^{2} v\left(x_{i}, t\right)=v^{q}\left(x_{i}, t\right) \int_{0}^{t} v^{p}\left(x_{i}, s\right) d s-\frac{h^{2}}{12} v_{x x x x}\left(\tilde{x}_{i}, t\right), 1 \leq i \leq I, \\
\text { for } t \in(0, t(h)) .
\end{gathered}
$$

Let $e_{h}(t)=V_{h}(t)-v_{h}(t)$ be the error of discretization. For the mean value theorem, we have for $1 \leq i \leq I, t \in(0, t(h))$,

$$
\frac{d e_{i}(t)}{d t}-\delta^{2} e_{i}(t)=q\left(\xi_{i}(t)\right)^{q-1} e_{i}(t) \int_{0}^{t} v^{p}\left(x_{i}, s\right) d s-V_{i}^{q}(t) \int_{0}^{t} p\left(\theta_{i}(s)\right)^{p-1} e_{i}(s) d s+K h^{2},
$$

where $\xi_{i}$ and $\theta_{i}$ are intermediate values between $V_{i}(t)$ and $v\left(x_{i}, t\right)$. Using (10) and (12), we deduce that, there exists a positive constant $L$ such that

$$
\frac{d e_{i}(t)}{d t}-\delta^{2} e_{i}(t) \leq L\left|e_{i}(t)\right|+L \int_{0}^{t}\left|e_{i}(s)\right| d s+K h^{2}, 1 \leq i \leq I, t \in(0, t(h)) .
$$

Introduce the vector $Y_{h}(t)$ defined as follows

$$
Y_{i}(t)=e^{L(1+T) t}\left(\left\|\varphi_{h}-v_{h}(0)\right\|_{\infty}+K h^{2}\right), 1 \leq i \leq I, t \in(0, t(h)) .
$$

A straightforward calculation reveals that

$$
\begin{gathered}
\frac{Y_{i}(t)}{d t}-\delta^{2} Y_{i}(t)>L\left|Y_{i}(t)\right|+L \int_{0}^{t}\left|Y_{i}(s)\right| d s+K h^{2}, 1 \leq i \leq I, t \in(0, t(h)), \\
Y_{0}(t)>e_{0}(t), t \in(0, t(h)), \\
Y_{i}(0)>e_{i}(0), 1 \leq i \leq I .
\end{gathered}
$$

It follows from comparison Lemma 2 that

$$
Y_{i}(t)>e_{i}(t) \text { for } 0 \leq i \leq I, t \in(0, t(h)) .
$$

By the same way, we also prove that

$$
Y_{i}(t)>-e_{i}(t) \text { for } 0 \leq i \leq I, t \in(0, t(h)),
$$

which implied that

$$
\left\|V_{h}(t)-v_{h}(t)\right\|_{\infty} \leq e^{L(1+T)}\left(\left\|\varphi_{h}-v_{h}(0)\right\|_{\infty}+K h^{2}\right) \text { for } t \in(0, t(h)) .
$$

Let us suppose that $t(h)<\min \left\{T, T_{b}^{h}\right\}$. From (11), we obtain

$$
1=\left\|V_{h}(t(h))-v_{h}(t(h))\right\|_{\infty} \leq e^{L(1+T)}\left(\left\|\varphi_{h}-v_{h}(0)\right\|_{\infty}+K h^{2}\right) .
$$

Since the third term of the above inequality goes to zero as $h$ goes to zero, we conclude that $1 \leq 0$, which is impossible. Consequently $t(h)=\min \left\{T, T_{b}^{h}\right\}$. Now let us show that $t(h)=T$. Suppose that $t(h)=T_{b}^{h}<T$.

Reasoning as above, we prove that we have a contradiction and the proof is complete. Now, we are in position to state the main theorem of this section.

Theorem 3 Suppose that the problem (1)-(3) has a solution $v$ which blows up in a finite time $T_{b}$ such that $v \in C^{4,1}([0,1] \times$ $\left.\left[0, T_{b}\right)\right)$ and the initial datum at (6) satisfies

$$
\left\|\varphi_{h}-v_{h}(0)\right\|_{\infty}=o(1) \text { as } h \rightarrow 0 .
$$


Under the hypothesis of Theorem 2, the problem (4)-(6) has a solution $V_{h}$ which blows up in a finite time $T_{b}^{h}$ and we have

$$
\lim _{h \rightarrow 0} T_{b}^{h}=T_{b} .
$$

Proof. Let $\epsilon>0$. There exists a positive constant $R$ such that

$$
\frac{2}{\pi^{2}} \ln \left(1-\frac{\pi^{2}}{2 A} e^{\frac{-\pi^{2}}{2} T} \frac{x^{1-q}}{1-q}\right) \leq \frac{\epsilon}{2} \text { for } x \in[R, \infty) .
$$

Since $v$ blows up in the time $T_{b}$, there exists a time $T_{0} \in\left(T_{b}-\frac{\epsilon}{2}, T_{b}\right)$ such that $\|v(., t)\|_{\infty} \geq 2 R$ for $t \in\left[T_{0}, T_{b}\right]$. Set $\frac{T_{0}+T_{b}}{2}$. From Theorem 2, the problem (4)-(6) has a solution $V_{h}(t)$ and we get

$$
\left\|V_{h}(t)-v_{h}(t)\right\|_{\infty} \leq R \text { for } x \in\left[0, T_{1}\right] .
$$

Applying the triangle inequality, we find that

$$
\left\|V_{h}\left(T_{1}\right)\right\|_{\infty} \geq\left\|v_{h}\left(T_{1}\right)\right\|_{\infty}-\left\|V_{h}\left(T_{1}\right)-v_{h}\left(T_{1}\right)\right\|_{\infty} \geq R .
$$

From Theorem 2, $V_{h}(t)$ blows up at the time $T_{b}^{h}$. We deduce from Remark 1 that

$$
\left|T_{b}^{h}-T_{1}\right| \leq \frac{2}{\pi^{2}} \ln \left(1-\frac{\pi^{2}}{2 A} e^{\frac{-\pi^{2}}{2} T_{1}} \frac{\left\|V_{h}\left(T_{1}\right)\right\|_{\infty}^{1-q}}{1-q}\right) \leq \frac{\epsilon}{2} .
$$

We deduce from (13) that

$$
\left|T_{b}^{h}-T_{b}\right| \leq\left|T_{b}^{h}-T_{1}\right|+\left|T_{1}-T_{b}\right| \leq \frac{\epsilon}{2}+\frac{\epsilon}{2} \leq \epsilon,
$$

which leads us to the desired result.

\section{Discretizations}

In this section, we study the phenomenon of blow-up using a discrete explicit scheme of (1)-(3). At first setting $f(x, t)=$ $\int_{0}^{t} v^{p}(x, s) d s$ we see that $f_{t}(x, t)=v^{p}(x, t)$. Therefore the problem (1)-(3) becomes

$$
\begin{gathered}
v_{t}=v_{x x}+(v(x, t))^{q} f(x, t), \quad x \in(0,1), \quad t \in(0, T), \\
f_{t}(x, t)=v^{p}(x, t), \quad x \in(0,1) \quad t \in(0, T), \\
v(0, t)=0, \quad v_{x}(1, t)=0, \quad t \in(0, T), \\
v(x, 0)=v_{0}(x)>0, \quad f(x, 0)=f_{0}(x), \quad x \in(0,1) .
\end{gathered}
$$

Approximate the solution $v(x, t)$ of (14)-(17) by the solution $V_{h}^{(n)}=\left(V_{0}^{(n)}, V_{1}^{(n)}, \ldots, V_{I}^{(n)}\right)^{T}$ of the following explicit scheme

$$
\begin{gathered}
\delta_{t} V_{i}^{(n)}=\delta^{2} V_{i}^{(n)}+\left(V_{i}^{(n)}\right)^{q} f\left(V_{i}^{(n)}, t_{n}\right), \quad 1 \leq i \leq I, \\
\delta_{t} f\left(V_{i}^{(n)}, t_{n}\right)=\left(V_{i}^{(n)}\right)^{p}, \quad 1 \leq i \leq I, \\
V_{0}^{(n)}=0, \\
V_{i}^{(0)}=\varphi_{i},
\end{gathered}
$$

where $n \geq 0, f\left(V_{i}^{(n)}, t_{n}\right)$ is the approximation of $\int_{0}^{t_{n}} v^{p}\left(x_{i}, s\right) d s$,

$$
\delta_{t} V_{i}^{(n)}=\frac{V_{i}^{(n+1)}-V_{i}^{(n)}}{\Delta t_{n}} \quad \text { and } \quad \delta_{t} f\left(V_{i}^{(n)}, t_{n}\right)=\frac{f\left(V_{i}^{(n+1)}, t_{n+1}\right)-f\left(V_{i}^{(n)}, t_{n}\right)}{\Delta t_{n}}
$$

with

$$
\Delta t_{n}=\min \left\{\frac{h^{2}}{3}, \tau\left\|V_{h}^{(n)}\right\|_{\infty}^{1-q}\right\}, \quad 0<\tau<1 .
$$

Let us notice that the restriction on the time step ensures the nonnegativity of the discrete solution. More precisely, one easily sees that $V_{i}^{(n)}>0, \quad 0 \leq i \leq I$. The following lemma is a discrete form of the maximum principle. 
Lemma 7 Let $a_{h}^{(n)}$ be a bounded vector and let $W_{h}^{(n)}$ a sequence such that

$$
\begin{gathered}
\delta_{t} W_{i}^{(n)}-\delta^{2} W_{i}^{(n)}+a_{i}^{(n)} W_{i}^{(n)} \geq 0, \quad 1 \leq i \leq I, \quad n \geq 0, \\
W_{0}^{(n)} \geq 0, \quad n \geq 0, \\
W_{i}^{(0)} \geq 0, \quad 0 \leq i \leq I .
\end{gathered}
$$

Then $W_{i}^{(n)} \geq 0$ for $0 \leq i \leq I, \quad n \geq 0$, if $\Delta t_{n} \leq \frac{h^{2}}{2+\left\|a_{h}^{(n)}\right\|_{\infty} h^{2}}$.

Proof. See (N'gohisse and Boni, 2011).

Lemma 8 Let $V_{h}^{(n)}$ be the solution of (18)-(21).

Then

$$
V_{i+1}^{(n)}>V_{i}^{(n)}, \quad 0 \leq i \leq I-1 .
$$

Proof. See (N'gohisse and Boni, 2011).

Lemma 9 Suppose that $a_{h}^{(n)}$ and $b_{h}^{(n)}$ are two vectors such that $a_{h}^{(n)}$ is bounded. Let $W_{h}^{(n)}$ and $X_{h}^{(n)}$ be two sequences such that

$$
\begin{gathered}
\delta_{t} W_{i}^{(n)}-\delta^{2} W_{i}^{(n)}+a_{i}^{(n)} W_{i}^{(n)}+b_{i}^{(n)} \leq \delta_{t} X_{i}^{(n)}-\delta^{2} X_{i}^{(n)}+a_{i}^{(n)} X_{i}^{(n)}, \quad 1 \leq i \leq I, \quad n \geq 0, \\
W_{0}^{(n)} \leq X_{0}^{(n)}, \quad n \geq 0, \\
W_{i}^{(0)} \leq X_{i}^{(0)}, \quad 0 \leq i \leq I .
\end{gathered}
$$

Then $W_{i}^{(n)} \leq X_{i}^{(n)}$ for $0 \leq i \leq I, \quad n \geq 0$, if $\Delta t_{n} \leq \frac{h^{2}}{2+\left\|a_{h}^{(n)}\right\|_{\infty} h^{2}}$.

Now, let us give a property of the operators $\delta_{t}$.

Lemma 10 Let $V^{(n)} \in \mathbb{R}$ be a sequence such that $V^{(n)} \geq 0$. Then we have

$$
\delta_{t}\left(V^{(n)}\right)^{q} \geq q\left(V^{(n)}\right)^{q-1} \delta_{t} V^{(n)}, \quad n \geq 0 .
$$

Proof. From Taylor's expansion, we find that

$$
\delta_{t}\left(V^{(n)}\right)^{q}=q\left(V^{(n)}\right)^{q-1} \delta_{t} V^{(n)}+\Delta t_{n} q(q-1)\left(\theta^{(n)}\right)^{q-2} \delta_{t}\left(V^{(n)}\right)^{2},
$$

where $\theta^{(n)}$ is an intermediate value between $V^{(n)}$ and $V^{(n+1)}$. Use the fact that $V^{(n)} \geq 0$ for $n \geq 0$ to complete the proof.

In order to treat the phenomenon of blow-up for discrete equations, we need the following definition.

Definition 1 We say that the solution $V_{h}^{(n)}$ of (18)-(21) blows up in a finite time if $\lim _{n \rightarrow+\infty}\left\|V_{h}^{(n)}\right\|_{\infty}=+\infty$ and the series $\sum_{n=0}^{\infty} \Delta t_{n}$ converges. The quantity $\sum_{n=0}^{\infty} \Delta t_{n}$ is called the numerical blow-up time of $V_{h}^{(n)}$.

The following theorem is the discrete version of Theorem 2 .

Theorem 3 Suppose that there exists a constant $A \in(0,1]$, such that the initial datum at (21) satisfies

$$
\delta^{2} \varphi_{i} \geq A \sin \left(i h \frac{\pi}{2}\right) \varphi_{i}^{q}, \quad 0 \leq i \leq I .
$$

Then the solution $V_{h}^{(n)}$ of (18)-(21) blows up in a finite time and its numerical blow-up time $T_{h}^{\Delta t}$ is estimated as follows

$$
T_{h}^{\Delta t} \leq \frac{\tau\left\|\varphi_{h}\right\|_{\infty}^{1-q}}{1-\left(1-\tau^{\prime}\right)^{1-q}} \quad \text { where } \quad \tau^{\prime}=A \min \left\{\frac{h^{2}}{3}\left\|\varphi_{h}\right\|_{\infty}^{q-1}, \tau\right\}
$$

Proof. Introduce the vector $J_{h}$ such that

$$
\begin{gathered}
J_{i}^{(n)}=\delta_{t} V_{i}^{(n)}-C_{i}^{(n)}\left(V_{i}^{(n)}\right)^{q}, \quad 0 \leq i \leq I, \\
\text { where } C_{i}^{(n)}=A e^{-\lambda_{h} \sum_{j=0}^{n-1} \Delta t_{j}} \sin \left(i h \frac{\pi}{2}\right), \text { with } \lambda_{h}=\frac{2-2 \cos \left(i h \frac{\pi}{2}\right)}{h^{2}} .
\end{gathered}
$$


A straightforward computation yields

$$
\begin{gathered}
\delta_{t} J_{i}^{(n)}-\delta^{2} J_{i}^{(n)}=\delta_{t}\left(\delta_{t} V_{i}^{(n)}-\delta^{2} V_{i}^{(n)}\right)-C_{i}^{(n)} \delta_{t}\left(V_{i}^{(n)}\right)^{q}+\delta^{2}\left(C_{i}^{(n)}\left(V_{i}^{(n)}\right)^{q}\right) \\
-\delta_{t} C_{i}^{(n)}\left(V_{i}^{(n)}\right)^{q}, \quad 1 \leq i \leq I .
\end{gathered}
$$

Using (18), we arrive at

$$
\delta_{t} J_{i}^{(n)}-\delta^{2} J_{i}^{(n)}=\delta_{t}\left(V_{i}^{q} f\left(V_{i}^{(n)}, t\right)\right)-C_{i}^{(n)} \delta_{t}\left(V_{i}^{(n)}\right)^{q}+\delta^{2}\left(C_{i}^{(n)}\left(V_{i}^{(n)}\right)^{q}\right)-\delta_{t} C_{i}^{(n)}\left(V_{i}^{(n)}\right)^{q} .
$$

From Lemmas 5 and 6, we get

$$
\delta^{2}\left(C_{i}^{(n)}\left(V_{i}^{q}\right) \geq C_{i}^{(n)} q V_{i}^{q-1} \delta^{2} V_{i}^{(n)}+V_{i}^{q} \delta^{2} C_{i}^{(n)}, \quad 1 \leq i \leq I .\right.
$$

Using the above estimates and Lemma 4, we discover that

$$
\begin{gathered}
\delta_{t} J_{i}^{(n)}-\delta^{2} J_{i}^{(n)} \geq \delta_{t}\left(V_{i}^{q} f\left(V_{i}^{(n)}, t\right)\right)-C_{i}^{(n)} q\left(V_{i}^{(n)}\right)^{q-1} \delta_{t} V_{i}^{(n)}+C_{i}^{(n)} q V_{i}^{q-1} \delta^{2} V_{i}^{(n)} \\
-V_{i}^{q}\left(\delta_{t} C_{i}^{(n)}-\delta^{2} C_{i}^{(n)}\right), \quad 1 \leq i \leq I .
\end{gathered}
$$

We observe that

$$
\delta_{t} C_{i}-\delta^{2} C_{i} \leq 0, \quad C_{i-1}<C_{i}, \quad 1 \leq i \leq I .
$$

Taking into account (18), we deduce that

$$
\delta_{t} J_{i}^{(n)}-\delta^{2} J_{i}^{(n)} \geq \delta_{t}\left(V_{i}^{q} f\left(V_{i}^{(n)}, t_{n}\right)\right)-q V_{i}^{q-1} C_{i}^{(n)}\left(V_{i}^{q} f\left(V_{i}^{(n)}, t_{n}\right)\right) .
$$

Using the fact that $\delta_{t}\left(f^{(n)}\left(V_{i}^{(n)}\right)^{q}\right)=f^{(n)} \delta_{t}\left(V_{i}^{(n)}\right)^{q}+\left(V_{i}^{(n)}\right)^{q} \delta_{t} f_{i}^{(n)}$ we arrive at

$$
\begin{gathered}
\delta_{t} J_{i}^{(n)}-\delta^{2} J_{i}^{(n)} \geq f^{(n)} \delta_{t}\left(V_{i}^{(n)}\right)^{q}+\left(V_{i}^{(n)}\right)^{p+q} \\
-C_{i}^{(n)} q\left(V_{i}^{(n)}\right)^{q-1}\left(V_{i}^{q} f\left(V_{i}^{(n)}, t_{n}\right)\right),
\end{gathered}
$$

which lead us

$$
\begin{gathered}
\delta_{t} J_{i}^{(n)}-\delta^{2} J_{i}^{(n)} \geq q\left(V_{i}^{(n)}\right)^{q-1} f\left(V_{i}^{(n)}, t_{n}\right) \delta_{t} V_{i}^{(n)}+\left(V_{i}^{(n)}\right)^{p+q} \\
-C_{i}^{(n)} q\left(V_{i}^{(n)}\right)^{q-1}\left(V_{i}^{q} f\left(V_{i}^{(n)}, t_{n}\right)\right), \quad 1 \leq i \leq I .
\end{gathered}
$$

Due to the fact that $\delta_{t} V_{i}^{(n)}=J_{i}^{(n)}+A\left(V_{i}^{(n)}\right)^{q}$, we arrive at

$$
\delta_{t} J_{i}^{(n)}-\delta^{2} J_{i}^{(n)} \geq\left(q\left(V_{i}^{(n)}\right)^{q-1} f\left(V_{i}^{(n)}, t_{n}\right)\right) J_{i}^{(n)}, \quad 1 \leq i \leq I .
$$

Obviously, we have $J_{0}^{(n)}=0$ and from (22), we obtain $J_{h}^{(0)} \geq 0$. It follows from Lemma 7 that $J_{h} \geq 0$. Hence, we have

$$
\frac{V_{i}^{(n+1)}-V_{i}^{(n)}}{\Delta t_{n}} \geq C_{i}^{(n)}\left(V_{i}^{(n)}\right)^{q}, \quad 0 \leq i \leq I .
$$

Consequently, we get

$$
V_{I}^{(n+1)} \geq V_{I}^{(n)}+C_{I}^{(n)} \Delta t_{n}\left(V_{I}^{(n)}\right)^{q}
$$

Since from Lemma $8, V_{I}^{(n)}=\left\|V_{h}^{(n)}\right\|_{\infty}$.

We arrive at

$$
\left\|V_{h}^{(n+1)}\right\|_{\infty} \geq\left\|V_{h}^{(n)}\right\|_{\infty}+C_{I}^{(n)} \Delta t_{n}\left\|V_{h}^{(n)}\right\|_{\infty}^{q} .
$$

We observe that

$$
\Delta t_{n}\left\|V_{h}^{(n)}\right\|_{\infty}^{q}=\min \left\{\frac{h^{3}}{3}\left\|V_{h}^{(n)}\right\|^{q}, \tau\right\} .
$$

The inequality (23) shows that the sequence $\left\|V_{h}^{(n)}\right\|_{\infty}$ is increasing. By induction we obtain $\left\|V_{h}^{(n)}\right\|_{\infty} \geq\left\|V_{h}^{(0)}\right\|_{\infty}=\left\|\varphi_{h}\right\|_{\infty}$. It follows that

$$
C_{I}^{(n)} \Delta t_{n}\left(\left\|V_{h}^{(n)}\right\|_{\infty}\right)^{q-1} \geq A \min \left\{\frac{h^{2}}{3}\left(\left\|\varphi_{h}\right\|_{\infty}\right)^{q-1}, \tau\right\}=\tau^{\prime} .
$$


Consequently, we have

$$
\left\|V_{h}^{(n+1)}\right\|_{\infty} \geq\left\|V_{h}^{(n)}\right\|_{\infty}\left(1+\tau^{\prime}\right) \quad n>0 .
$$

Using a recursion argument, we discover that

$$
\left\|V_{h}^{(n)}\right\|_{\infty} \geq\left\|V_{h}^{(0)}\right\|_{\infty}\left(1+\tau^{\prime}\right)^{n}=\left\|\varphi_{h}\right\|_{\infty}\left(1+\tau^{\prime}\right)^{n} .
$$

Hence, we see that $\left\|V_{h}^{(n)}\right\|_{\infty}$ goes to infinity as $n$ approaches infinity. Now let us estimate the numerical blow-up time. From the restriction on the time step, we get

$$
T_{h}^{\Delta t}=\sum_{n=0}^{\infty} \Delta t_{n} \leq \sum_{n=0}^{+\infty} \tau\left(\left\|V_{h}^{(n)}\right\|_{\infty}\right)^{1-q} .
$$

Due to (25), we arrive at

$$
\sum_{n=0}^{\infty} \Delta t_{n} \leq \sum_{n=0}^{+\infty} \tau\left\|\varphi_{h}\right\|_{\infty}^{1-q}\left[\left(1+\tau^{\prime}\right)^{1-q}\right]^{n}
$$

Use the fact that the quantity on the right hand side of the above inequality converges toward $\frac{\tau \mid \varphi_{h} h_{\infty}^{1}-q}{\left[1-\left(1+\tau^{\prime}\right)^{1-q}\right]}$ to complete the rest of the proof.

Remark 2 From (24), we get by induction that

$$
\left\|V_{h}^{(n)}\right\|_{\infty} \geq\left\|V_{h}^{(k)}\right\|_{\infty}\left(1+\tau^{\prime}\right)^{n-k} \text { for } n \geq k
$$

Hence

$$
T_{h}^{\Delta t}-t_{k}=\sum_{n=q}^{+\infty} \Delta t_{n} \leq \sum_{n=0}^{+\infty} \tau\left\|V_{h}^{(k)}\right\|_{\infty}^{1-q}\left[\left(1+\tau^{\prime}\right)^{1-q}\right]^{n-k}
$$

We observe that

$$
T_{h}^{\Delta t}-t_{k} \leq \frac{\tau\left\|V_{h}^{(k)}\right\|_{\infty}^{1-q}}{1-\left(1+\tau^{\prime}\right)^{1-q}}
$$

when $h$ tends to zero. Since $\tau^{\prime}=\min \left\{\frac{h^{2}}{3}\left(\left\|\varphi_{h}\right\|_{\infty}\right)^{q-1}, \tau\right\}$, if we take $\tau=h^{2}$, we get $\frac{\tau}{\tau^{\prime}}=\min \left\{\frac{1}{3}\left(\left\|\varphi_{h}\right\|_{\infty}\right)^{q-1}, 1\right\}$ which implies that there exists a positive constant $K$ such that $\frac{\tau}{\tau^{\prime}} \leq K$.

The following theorem is the discrete form of Theorem 2.

Theorem 4 Suppose that the problem (14)-(17) has a solution $v \in C^{4,2}([0,1] \times[0, T])$. Assume that the initial datum at (21) verifies

$$
\left\|\varphi_{h}-v_{h}(0)\right\|_{\infty}=o(1) \quad \text { as } \quad h \rightarrow 0 .
$$

Then the problem (18)-(21) has a solution $V_{h}^{(n)}$ for $h$ sufficiently small,

$0 \leq n \leq J$ and we have the following estimate

$$
\max _{0 \leq n \leq J}\left\|V_{h}^{(n)}-v_{h}\left(t_{n}\right)\right\|_{\infty}=O\left(\left\|\varphi_{h}-v_{h}(0)\right\|_{\infty}+h^{2}+\Delta t_{n}\right) \quad \text { as } \quad h \rightarrow 0,
$$

where $J$ is such that $\sum_{n=0}^{J-1} \Delta t_{n} \leq T$ and $t_{n}=\sum_{j=0}^{n-1} \Delta t_{j}$.

Proof. For each $h$, the problem (18)-(21) has a solution $V_{h}^{(n)}$. Let $N \leq J$ be the greatest value of $n$ such that

$$
\left\|V_{h}^{(n)}-v_{h}\left(t_{n}\right)\right\|_{\infty}<1 \text { for } n<N .
$$

We know that $N \geq 1$ because of (26). The fact that $v \in C^{4,2}$, there exists a positive constant $\alpha$ such that $\|v\|_{\infty} \leq \alpha$. Applying the triangle inequality, we obtain

$$
\left\|V_{h}^{(n)}\right\|_{\infty} \leq\left\|v_{h}\left(t_{n}\right)\right\|_{\infty}+\left\|V_{h}^{(n)}-v_{h}\left(t_{n}\right)\right\|_{\infty} \leq 1+\alpha \text { for } n<N .
$$

As in the proof of Theorem 2, using Taylor's expansion, we find that

$$
\begin{aligned}
& \delta_{t} v\left(x_{i}, t_{n}\right)-\delta^{2} v\left(x_{i}, t_{n}\right)-\left(v\left(x_{i}, t_{n}\right)\right)^{q} f\left(x_{i}, t_{n}\right) \\
= & -\frac{h^{2}}{12} v_{x x x x}\left(\widetilde{x_{i}}, t_{n}\right)+\frac{\Delta t_{n}}{2} v_{t t}\left(x_{i}, \widetilde{t_{n}}\right), \quad 1 \leq i \leq I .
\end{aligned}
$$


Let $e_{h}^{(n)}=V_{h}^{(n)}-v_{h}\left(t_{n}\right)$ be the error of discretization. From the mean value theorem, we get for $n<N$,

$$
\begin{gathered}
\delta_{t} e_{i}^{(n)}-\delta^{2} e_{i}^{(n)}=q\left(s_{i}^{(n)}\right)^{q-1} e_{i}^{(n)} f\left(e_{i}^{(n)}, t_{n}\right)+v\left(x_{i}, t_{n}\right)^{q} \int_{0}^{t_{n}} \theta_{i}^{p-1}(s) e_{i}^{(n)} d s \\
+\frac{h^{2}}{12} v_{x x x x}\left(\widetilde{x}_{i}, t_{n}\right)-\frac{\Delta t_{n}}{2} v_{t t}\left(x_{i}, \widetilde{t}_{n}\right), \quad 1 \leq i \leq I,
\end{gathered}
$$

where $\varsigma_{i}^{(n)}$ and $\theta_{i}$ are intermediate values between $V_{i}^{(n)}$ and $v\left(x_{i}, t_{n}\right)$. Since $v_{x x x x}(x, t), v_{t t}(x, t)$ are bounded, and use (28) we deduce that, there exist some positives constants $M$ and $K$ such that

$$
\delta_{t} e_{i}^{(n)}-\delta^{2} e_{i}^{(n)} \leq K\left|e_{i}^{(n)}\right|+M \Delta t_{n}+M h^{2}, \quad 1 \leq i \leq I,
$$

where $K=1+\alpha$. Introduce the vector $W_{h}^{(n)}$ defined as follows

$$
W_{i}^{(n)}=e^{(K+1) t_{n}}\left(\left\|\varphi_{h}-v_{h}(0)\right\|_{\infty}+M \Delta t_{n}+M h^{2}\right), \quad 1 \leq i \leq I .
$$

A straightforward computation gives

$$
\begin{gathered}
\delta_{t} W_{i}^{(n)}-\delta^{2} W_{i}^{(n)} \geq K W_{i}^{(n)}+M \Delta t_{n}+M h^{2} \quad 1 \leq i \leq I, \\
W_{0}^{(n)} \geq e_{0}^{(n)} \\
W_{i}^{(0)} \geq e_{i}^{(0)}, \quad 1 \leq i \leq I .
\end{gathered}
$$

It follows from Comparison Lemma 9 that $W_{h}^{(n)} \geq e_{h}^{(n)}$. By the same way, we also prove that $W_{h}^{(n)} \geq-e_{h}^{(n)}$, which implies that

$$
\left\|W_{h}^{(n)}-v_{h}\left(t_{n}\right)\right\|_{\infty} \leq e^{(K+1) t_{n}}\left(\left\|\varphi_{h}-v_{h}(0)\right\|_{\infty}+M \Delta t_{n}+M h^{2}\right)
$$

Let us show that $N=J$. Suppose that $N<J$. If we replace $n$ by $N$ in the above inequality and use (27), we find that

$$
1 \leq\left\|V_{h}^{(N)}-v_{h}\left(t_{N}\right)\right\|_{\infty} \leq e^{(K+1) t_{N}}\left(\left\|\varphi_{h}-v_{h}(0)\right\|_{\infty}+M \Delta t_{n}+M h^{2}\right) .
$$

Since the term on the right hand side of the second inequality goes to zero as $h$ tends to zero, we deduce that $1 \leq 0$, which is a contradiction and the proof is complete.

Now, we are in position to prove the main theorem of this section.

Theorem 5 Suppose that the problem (14)-(17) has a solution $v$ which blows up in a finite time $T_{0}$ and $v \in C^{4,2}([0,1] \times$ $\left.\left[0, T_{0}\right)\right)$. Assume that the initial datum at (21) satisfies

$$
\left\|\varphi_{h}-v_{h}(0)\right\|_{\infty}=o(1) \quad \text { as } \quad h \rightarrow 0 .
$$

Under the assumption of Theorem 3, the problem (18)-(21) has a solution $V_{h}^{(n)}$ which blows up in a finite time $T_{h}^{\Delta t}$ and the following relation holds

$$
\lim _{h \rightarrow 0} T_{h}^{\Delta t}=T_{0} .
$$

Proof. Letting $\varepsilon>0$, there exists a constant $R>0$ such that

$$
\frac{\tau x^{1-q}}{1-\left(1+\tau^{\prime}\right)^{1-q}}<\frac{\varepsilon}{2} \quad \text { for } \quad x \in[R, \infty) .
$$

Since $v$ blows up at the time $T_{0}$, there exists $T_{1} \in\left(T_{0}-\frac{\varepsilon}{2}, T_{0}\right)$ such that

$$
\|v(\cdot, t)\|_{\infty} \geq 2 R \text { for } t \in\left[T_{1}, T_{0}\right) .
$$

Let $T_{2}=\frac{T_{1}+T_{0}}{2}$ and $k$ be a positive integer such that $t_{k}=\sum_{n=0}^{k-1} \Delta t_{n} \in\left[T_{1}, T_{2}\right]$ for $h$ small enough. We have $\sup _{t \in\left[0, T_{2}\right]}\|v(\cdot, t)\|_{\infty}<$ $\infty$. It follows from Theorem 4 that the problem (18)-(21) has a solution $V_{h}^{(n)}$ which obeys to

$$
\left\|V_{h}^{(n)}-v_{h}\left(t_{n}\right)\right\|_{\infty}<R \quad \text { for } n \leq k
$$

which implies that

$$
\left\|V_{h}^{(k)}\right\|_{\infty} \geq\left\|v_{h}\left(t_{k}\right)\right\|_{\infty}-\left\|V_{h}^{(k)}-v_{h}\left(t_{k}\right)\right\|_{\infty} \geq R
$$


From Theorem 3, $V_{h}^{(n)}$ blows up at the time $T_{h}^{\Delta t}$. It follows from Remark 2 and (29) that

$$
\left|T_{h}^{\Delta t}-t_{k}\right| \leq \frac{\tau\left\|V_{h}^{(k)}\right\|_{\infty}^{1-q}}{1-\left(1+\tau^{\prime}\right)^{1-q}}<\frac{\varepsilon}{2},
$$

because $\left\|V_{h}^{(k)}\right\|_{\infty} \geq R$. We deduce that

$$
\left|T_{0}-T_{h}^{\Delta t}\right| \leq\left|T_{0}-t_{k}\right|+\left|t_{k}-T_{h}^{\Delta t}\right| \leq \frac{\varepsilon}{2}+\frac{\varepsilon}{2} \leq \varepsilon,
$$

and the proof is complete.

\section{Numerical Results}

In this section, we present some numerical approximations of the blow-up time for the solution of the problem (1)-(3) in the case where $v_{0}(x)=10 \sin (\pi x)$. Firstly, we consider the explicit scheme in (18)-(21). Secondly, we use the following implicit scheme

$$
\begin{gathered}
\frac{V_{i}^{(n+1)}-V_{i}^{(n)}}{\Delta t_{n}}=\delta^{2} V_{i}^{(n+1)}+\left(V_{i}^{(n)}\right)^{q} f_{i}^{(n+1)}, \quad 1 \leq i \leq I, \\
\frac{f_{i}^{(n+1)}-f_{i}^{(n)}}{\Delta t_{n}}=\left(V_{i}^{(n)}\right)^{p}, \quad 1 \leq i \leq I, \\
\quad V_{0}^{(n)}=0, \\
V_{i}^{(0)}=\varphi_{i} \geq 0, \quad f_{i}^{(0)}=\psi_{i}, \quad 0 \leq i \leq I,
\end{gathered}
$$

where $n \geq 0$,

$$
\Delta t_{n}=\min \left\{\frac{h^{2}}{2}, \tau\left\|V_{h}^{(n)}\right\|_{\infty}^{1-q}\right\}, \quad 0<\tau<1
$$

In both cases, we take $\varphi_{i}=10 \sin \left(\frac{i \pi h}{2}\right), 0 \leq i \leq I$. For the above implicit scheme, the nonnegativity of the solution $V_{h}^{(n)}$ is guaranteed using standard methods see (Boni, 2001). In the tables 1, 2, 3 and 4, in rows, we present the numerical blow-up times, the numbers of iterations, the CPU times and the orders of the approximations corresponding to meshes of $16,32,64,128,256,512$. We take for the numerical blow-up time $t_{n}=\sum_{j=0}^{n-1} \Delta t_{j}$ which is computed at the first time when $\Delta t_{n}=\left|t_{n+1}-t_{n}\right| \leq 10^{-16}$. The order(s) of the method is computed from

$$
s=\frac{\log \left(\left(T_{4 h}-T_{2 h}\right) /\left(T_{2 h}-T_{h}\right)\right)}{\log (2)} .
$$

Table 1. Explicit Euler method for $p=1, q=2$

\begin{tabular}{lcccc}
\hline $\mathrm{I}$ & $t_{n}$ & $\mathrm{n}$ & CPUt & $\mathrm{s}$ \\
\hline 16 & 0.16258655 & 1962 & - & - \\
32 & 0.16231478 & 7669 & 1 & - \\
64 & 0.16224740 & 29937 & 2 & 2.0120 \\
128 & 0.16223066 & 116714 & 18 & 2.0090 \\
256 & 0.16222649 & 454334 & 1241 & 2.0052 \\
512 & 0.16222545 & 1765598 & 20027 & 2.0035 \\
\hline
\end{tabular}

Table 2. Implicit Euler method for $p=1, q=2$

\begin{tabular}{lcccc}
\hline $\mathrm{I}$ & $t_{n}$ & $\mathrm{n}$ & CPUt & $\mathrm{s}$ \\
\hline 16 & 0.16249945 & 1962 & - & - \\
32 & 0.16229298 & 7669 & - & - \\
64 & 0.16224195 & 29937 & 5 & 2.0165 \\
128 & 0.16222929 & 116714 & 129 & 2.0111 \\
256 & 0.16222615 & 454334 & 3442 & 2.0114 \\
512 & 0.16222537 & 1765598 & 71152 & 2.0092 \\
\hline
\end{tabular}


Table 3. Explicit Euler method for $p=0.5, q=1.5$

\begin{tabular}{lcccc}
\hline $\mathrm{I}$ & $t_{n}$ & $\mathrm{n}$ & $C P U t$ & $\mathrm{~s}$ \\
\hline 16 & 1.10140601 & 2918 & - & - \\
32 & 1.09866219 & 11390 & 1 & - \\
64 & 1.09797752 & 44520 & 5 & 2.0027 \\
128 & 1.09780647 & 173911 & 53 & 2.0010 \\
256 & 1.09776373 & 678617 & 986 & 2.0008 \\
512 & 1.09775266 & 2000001 & 59453 & 1.9489 \\
\hline
\end{tabular}

Table 4. Implicit Euler method for $p=0.5, q=1.5$

\begin{tabular}{lcccc}
\hline $\mathrm{I}$ & $t_{n}$ & $\mathrm{n}$ & $C P U t$ & $\mathrm{~s}$ \\
\hline 16 & 1.09643911 & 2918 & - & - \\
32 & 1.09741620 & 11390 & 1 & - \\
64 & 1.09766575 & 44519 & 15 & 1.9692 \\
128 & 1.09772851 & 173911 & 193 & 1.9914 \\
256 & 1.09774424 & 678617 & 3547 & 1.9963 \\
512 & 1.09774817 & 2644528 & 122749 & 2.0009 \\
\hline
\end{tabular}

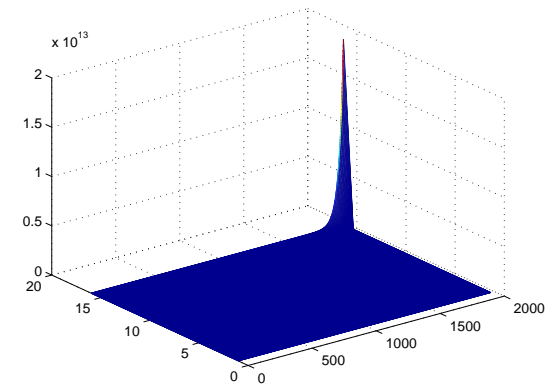

Figure 1. Evolution of discrete solution for $p=1, q=2$

Remark 3 From the above tables, we illustrate the convergence of the blow-up time of the solution of the problem (1)-(3) to the numerical one because the order of approximations of the method goes to 2, which is the accuracy of the difference approximation in space.

If we compare tables 1, 2 and tables 3, 4 we notice that the blow-up time depends strongly on the reaction term. In tables 1 and 2 when $p=1$ and $q=2$, we observe that the blow-up time is approximately equal to 0.1622 . In tables 3 and 4 when $p=0.5$ and $q=1.5$, the blow-up time is approximately equal to 1.0977 .

We can deduce that when the parameter $p$ tend to 0 and $q$ tend to 1 , it is difficult to obtain the phenomenom of blow-up, and the blow-up time is big enough.

\section{References}

Abia, L. M., Lopes-Marcos, J. C., \& Martinez, J. (1998). On the Blow-up time convergence of semidicretizations of reaction-diffusion equations. Appl. Numer. Math., 26, 399-414. https://doi.org/10.1016/S0168-9274(97)00105-0

Boni, T. K. (2001). Extinction for discretizations of some semilinear parabolic equations. C. R. Acad. Sci. Paris, Srie I, Math., 333, 795-800.

Brandle, C., Groisman, P., \& Rossi, J. D. (2004). Fully discrete adaptative methods for a blow-up problem. Math. Models Methods. Appl. Sci., 14, 1425-1450. https://doi.org/10.1142/S0218202504003751

Brandle, C., Quiros, F., \& Rossi, J. D. (2005). An adaptative numerical method to handle blow-up in a parabolic system. Numer. Maths., 102, 39-59. https://doi.org/10.1007/s00211-005-0638-X

Ferreira, R., Groisman, P., \& Rossi, J. D. (2004). Numerical blow-up for the porous medium equation with a source. Numer. Methods PDE, 20, 552-575. https://doi.org/10.1002/num.10103

Galaktionov, V. A., \& Vazquez, J. L. (2002). The problem of blow-up in nonlinear parabolic equation. Current developments in PDE(Temusco,1999) Dis. Conti. Syst. A., 8, 399-433. https://doi.org/10.3934/dcds.2002.8.399 
Groisman, P. (2006). Totaly discrete explicit and semi-explicit Euler method for a blow-up problem in several space dimension. Computing, 76, 325-352. https://doi.org/10.1007/s00607-005-0136-0

Groisman, P., \& Rossi, J. D. (2004). Dependance of the blow-up time with respect to parameters and numerical approximations for a parabolic problem. Asymptot. Anal, 37, 79-91.

Hirata, D. (1999). Blow-up for a class of semilinear integro-differential equations of parabolic type. Math. Methods Appl. Sci., 22, 1087-1100.

Koffi, N., \& Nabongo, D. (2016). Numerical blow-up for solutions of semilinear heat equations with small diffusion. Ann. Univ. Orader Fasc. Math, Tome XXIII, 21-36.

Kozhanov, A. (1994). Parabolic equations with nonlocal nonlinear source. Siberian Math., 35, 545-556. https://doi.org/10.1002/(SICI)/1099-1476

Li, F. S. (2009). Global existence and uniqueness of weak solution to non linear viscoelastic full Marguerre-von Karman shallows equations. Acta Math. Sin., 25, 2133-2156. https://doi.org/10.1007/s00033-003-1128-6.

Li, Y. X., \& Xie, C. H. (2004). Blow-up for semilinear parabolic equation with nonlinear memory. Z. Angew. Math. Phys, 55, 15-27. https://doi.org/10.1007\%2FBF02104572

Mai, I., \& Mochizuki, K. (1991). On blow-up of solutions for quasilinear degenerate parabolic equations. Publ. Res. Inst. Math. Sci., 27, 695-709.https://doi.org/10.2977/prims/1195169267

N'gohisse, F. K., \& Boni, T. K. (2008). Numerical Blow-up solution for some semilinear heat equation. Elect. Trans. of Numer. Analysis, 30, 247-258.

N'gohisse, F. K., \& Boni. T. K. (2011). Numerical blow-up for a nonlinear heat equation. Acta Math. Sin. English Serie, 27, 845-862. https://doi.org/10.1007/s10114-009-7048-4.

Pablo, A. D., Llanos, M., \& Ferreira, R. (2005). Numerical blow-up for p-Laplacien equation with a nonlinear source. Proceeding of equatdiff., 11, 363-367. https://doi.org/10.12478/cnam-2005-0007

Quittner, P., \& Souplet, P. (2007). Superlinear parabolic problems, Blow-up, Global existence and Steady States Series. Birkhuser Advanced Tests/ Basler Lehrbcher. https://doi.org/10.1007/978-37643-8442-5

Sobo, L. B. B., Yoro, G., \& Nachid, H. (2016). On asymptotic of the blow-up for differential equation in a large domain. Int. J. of Rec. S. Reas., 7, 12158-12168.

Souplet, P. (2004). Monotonicity of solution and blow-up for semilinear parabolic equation with nonlinear memory. $Z$. Angew. Math. Phys., 55, 28-31. https://doi.org/10.1007/s00033-003-1158-0

Zhang, L., Ziang, Y. S., \& Zhou, Z. (2010). Parabolic equation with VMO coefficients in generalized Merrey Morrey. Acta. Sin., 26, 117-130.

Zhou, J. (2007). Non-similitaneous blow-up for a semilinear parabolic system with nonlinear memory. Survey in Math. and Appl., 2, 21-27.

\section{Copyrights}

Copyright for this article is retained by the author(s), with first publication rights granted to the journal.

This is an open-access article distributed under the terms and conditions of the Creative Commons Attribution license (http://creativecommons.org/licenses/by/4.0/). 